\title{
Electromagnetic waves scattering from a sphere of complex conjugate medium
}

\author{
Ahsan Illahi ${ }^{1}$, M. Bashir ${ }^{2}$, Majeed A. S. Alkanhal ${ }^{3}$, Sadia Khatoon ${ }^{1}$, Abdul Ghaffar ${ }^{4^{*}}$ and Y. Khan ${ }^{3}$
}

\begin{abstract}
A boundary value problem involving the scattering of electromagnetic waves from a sphere of complex conjugate medium (CCM) is studied. The sphere is placed in free space. The source of excitation for the sphere in our case is a plane wave. Incident, scattered and transmitted fields are formulated. The unknown coefficients in the scattered and transmitted fields are found using boundary conditions. From these electromagnetic fields, the Mie efficiencies are determined. The technique used in studying the scattering of electromagnetic waves from CCM is analytical and a closed form solution is obtained. It is shown by numerical results that the scattering is enhanced in case of CCM sphere as a target. Results for the limiting cases are also derived to compare the validity of our formulation with the published work.
\end{abstract}

Keywords: Scattering, Sphere, Complex medium

\section{Introduction}

The complex refractive index $N=n \pm i k$ having real and imaginary parts are very important in dealing with the intrinsic optical properties. Complex conjugate medium is a special type of medium which is lossless medium having real value of refractive index. The concept of complex conjugate medium (CCM) was introduced a few years ago. Dragoman [1] proposed this medium; the reflection and transmission properties of this medium was discussed in detail by him. The CCM has complex value of relative electric permittivity and relative magnetic permeability and real refractive index i.e.

$$
\begin{aligned}
& \varepsilon_{r}=m(a+i b) \\
& \mu_{r}=(a-i b)
\end{aligned}
$$

and refractive index $N=\sqrt{\varepsilon_{r} \mu_{r}}=\sqrt{m\left(a^{2}+b^{2}\right)}$, where ' $m$ ' is any positive integer.

The CCM modifies the scattering properties $[2,3]$ and no energy absorption losses occur. The attenuation in the electromagnetic wave through the CCM medium is only due to its refractive index and absorption losses are zero [4]. Absorption is determined by the imaginary

\footnotetext{
* Correspondence: aghaffar16@uaf.edu.pk

${ }^{4}$ Department of Physics, University of Agriculture Faisalabad, Faisalabad,

Pakistan

Full list of author information is available at the end of the article
}

parts of these two parameters (i.e. $\varepsilon_{r}$ and $\mu_{r}$ ). The attenuation of electromagnetic wave is determined or controlled by the imaginary part while the real part determines the phase velocity of waves as it traverses through the medium.

CCM is electrically active and magnetically dissipative or electrically dissipative and magnetically active. The gain coefficient in optical active materials (e.g. in the non-impurified non-magnetic semiconductor nano-sized lasers) is controlled by injection current. It can be tuned to the value required by CCM when $\mathrm{Mn}$ is used as dopant. Thus, CCM can be realized using the existing technology [1].

Scattering theory is a very important tool of physics. It provides important information about the particles or obstacles $[5,6]$. Scattering of electromagnetic waves by spherical objects is very interesting and carry many applications $[7,8]$. Sphere is one of the few three-dimensional bounded geometrical shapes that possess a closed form analytical solution [9]. It is commonly used as a reference for any kind of electromagnetic scattering theory and applications. Complete analytical treatment of electromagnetic plane wave scattering by a homogeneous, isotropic, dielectric sphere can be traced back to an 1890 paper by Lorenz, though credit for that achievement is commonly given to 1908 paper by Mie [10] . In this study, the scattering of plane waves from CCM sphere is considered. The 
Mie efficiencies i.e. extinction, scattered, absorption and backscattered efficiencies have been computed.

This work will demonstrate that the scattering efficiencies in case of CCM sphere dominates over the conventional dielectric sphere. This strong scattering effect can enhance significantly the light absorption in thin films and thus power conversion efficiencies of related solar cells which has already been investigated for dielectric nanospheres by several researchers e. g. [11] and experimentally demonstrated by [12]. This study related to the CCM sphere has not yet been reported before to the best of our knowledge.

\section{Methods and formulation}

Consider a uniform plane wave with frequency $\omega$ propagating along positive $z$ axis, from one medium to another medium. Let it travels from medium 1 i.e., space which is characterized by $\varepsilon_{0}$ and $\boldsymbol{\mu}_{\mathbf{0}}$ to a linear, isotropic and homogenous medium 2 i.e., CCM having relative electric permittivity $\boldsymbol{\varepsilon}_{\boldsymbol{r}}$ and relative magnetic permeability $\boldsymbol{\mu}_{r}$ (Fig. 1).

The incident fields can be written as [13]

$$
\begin{aligned}
\mathbf{E}_{i} & =E_{0} e^{-j k_{2} z+j \omega t} \boldsymbol{a}_{x} \\
& =E_{0} e^{j \omega t} \sum_{n=1}^{\infty}(-j)^{n} \frac{2 n+1}{n(n+1)}\left(\mathbf{m}_{01 n}^{(1)}-i \mathbf{n}_{e 1 n}^{(1)}\right)
\end{aligned}
$$

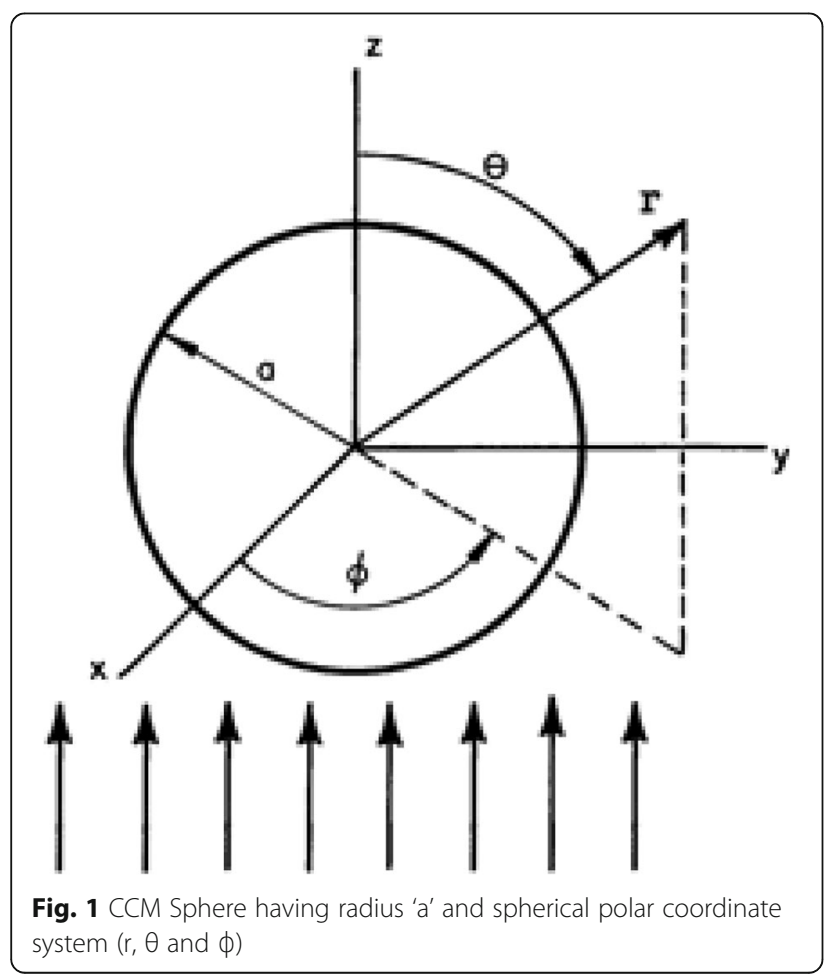

$$
\begin{aligned}
\mathbf{H}_{i} & =E_{0} \frac{k_{2}}{\mu_{2} \omega} e^{-j k_{2} z+j \omega t} \boldsymbol{a}_{y} \\
& =-\frac{k_{2}}{\mu_{2} \omega} E_{0} e^{j \omega t} \sum_{n=1}^{\infty}(-j)^{n} \frac{2 n+1}{n(n+1)}\left(\mathbf{m}_{e 1 n}^{(1)}+i \mathbf{n}_{01 n}^{(1)}\right)
\end{aligned}
$$

where, $E_{0}$ is the amplitude of the incident wave, and

$\mathbf{m}_{o 01 n}^{(1)}= \pm \frac{1}{\sin \theta} j_{n}\left(k_{2} R\right) P_{n}^{1}(\cos \theta){ }_{\sin }^{\cos } \phi \mathbf{i}_{2}-j_{n}\left(k_{2} R\right) \frac{\partial}{\partial \theta} P_{n}^{1} \cos _{\cos }^{\sin } \phi \mathbf{i}_{3}$

here, subscripts 'o' and ' $e$ ' are for odd and even values, respectively, and $J_{n}$ is used for Bessel function.

$$
\begin{aligned}
\boldsymbol{n}_{e 01 n}^{(1)} & =\frac{n(n+1)}{k_{2} R} j_{n}\left(k_{2} R\right) P_{n}^{1}(\cos \theta){ }_{\cos }^{\sin } \phi \boldsymbol{i}_{1} \\
& +\frac{1}{k_{2} R}\left[k_{2} R j_{n}\left(k_{2} R\right)\right]^{\prime} \frac{\partial}{\partial \theta} P_{n}^{1} \sin _{\cos }^{\sin } \phi \boldsymbol{i}_{2} \\
& \pm \frac{1}{k_{2} R \sin \theta}\left[k_{2} R j_{n}\left(k_{2} R\right)\left[k_{2} R j_{n}\left(k_{2} R\right)\right]^{\prime} P_{n}^{1}(\cos \theta){ }_{\sin }^{\cos } \phi \boldsymbol{i}_{3}\right.
\end{aligned}
$$

The prime sign denotes the differentiation with respect to $k_{2} R$. There are two parts of the induced secondary fields; first is transmitted field and the other is reflected field. The transmitted field only applies to interior of the sphere while reflected field extends up to infinity. We may write

$$
\begin{aligned}
& \boldsymbol{E}_{r}=E_{0} e^{j \omega t} \sum_{n=1}^{\infty}(-j)^{n} \frac{2 n+1}{n(n+1)}\left(a_{n}^{r} \boldsymbol{m}_{o 1 n}^{(3)}-i b_{n}^{r} \boldsymbol{n}_{e 1 n}^{(3)}\right) \\
& \boldsymbol{H}_{r}=-\frac{k_{2}}{\omega \mu_{2}} E_{0} e^{j \omega t} \sum_{n=1}^{\infty}(-j)^{n} \frac{2 n+1}{n(n+1)}\left(b_{n}^{r} \boldsymbol{m}_{e 1 n}^{(3)}-j a_{n}^{r} \boldsymbol{n}_{o 1 n}^{(3)}\right)
\end{aligned}
$$

which is valid for $R>a$, and

$$
\begin{aligned}
& \boldsymbol{E}_{t}=E_{0} e^{j \omega t} \sum_{n=1}^{\infty}(-j)^{n} \frac{2 n+1}{n(n+1)}\left(a_{n}^{t} \boldsymbol{m}_{o 1 n}^{(1)}+j b_{n}^{t} \boldsymbol{n}_{e 1 n}^{(1)}\right) \\
& \boldsymbol{H}_{t}=-\frac{k_{1}}{\omega \mu_{1}} E_{0} e^{j \omega t} \sum_{n=1}^{\infty}(-j)^{n} \frac{2 n+1}{n(n+1)}\left(b_{n}^{t} \boldsymbol{m}_{e 1 n}^{(1)}-j a_{n}^{t} \boldsymbol{n}_{o 1 n}^{(1)}\right)
\end{aligned}
$$

which is applicable for $R<a$. the functions $\boldsymbol{m}_{e 01 n}^{(1)}$ and $\boldsymbol{n}_{e 01 n}^{(1)}$ are deduced by replacing $j_{n}\left(k_{2} R\right)$ by $h_{n}^{1}\left(k_{2} R\right)$

\section{Boundary Conditions}

On the surface of CCM sphere, both the electric and magnetic field components are continuous. i.e. at $R=a$, 


$$
i_{1} \times\left(E_{i}-E_{r}\right)=i_{1} \times E_{t}
$$

These B.Cs, leads to two pairs of equations for expansion coefficients:

$$
\begin{gathered}
a_{n}^{t} j_{n}(N \rho)-a_{n}^{r} h_{n}^{(1)}(\rho)=j_{n}(\rho) \\
\mu_{2} a_{n}^{t}\left[N \rho j_{n}(N \rho)\right]^{\prime}-\mu_{1} a_{n}^{r}\left[\rho h_{n}^{1}(\rho)\right]^{\prime}=\mu_{1}\left[\rho j_{n}(\rho)\right]^{\prime} \\
\mu_{2} N b_{n}^{t} j_{n}(N \rho)-\mu_{1} b_{n}^{r} h_{n}^{(1)}(\rho)=\mu_{1} j_{n}(\rho) \\
b_{n}^{t}\left[N \rho j_{n}(N \rho)\right]^{\prime}-N b_{n}^{r}\left[\rho h_{n}^{(1)}(\rho)\right]^{\prime}=N\left[\rho j_{n}(\rho)\right]^{\prime}
\end{gathered}
$$

where,

$$
k_{1}=N k_{2} \rho=k_{2} a k_{1} a=N \rho
$$

Now, the coefficients of external field are given by,

$$
\begin{gathered}
a_{n}^{r}=-\frac{\mu_{1} j_{n} N(\rho)\left[\rho j_{n}(\rho)\right]^{\prime}-\mu_{2} j_{n}(\rho)\left[N \rho j_{n}(N \rho)\right]^{\prime}}{\mu_{1} j_{n}(N \rho)\left[\rho h_{n}^{(1)}(\rho)\right]^{\prime}-\mu_{2} h_{n}^{(1)}(\rho)\left[N \rho j_{n}(N \rho)\right]^{\prime}} \\
b_{n}^{r}=-\frac{\mu_{1} j_{n}(\rho)\left[N \rho j_{n}(N \rho)\right]^{\prime}-\mu_{2} N^{2} j_{n}(N \rho)\left[\rho j_{n}(\rho)\right]^{\prime}}{\mu_{1} h_{n}^{(1)}(\rho)\left[N \rho j_{n}(N \rho)\right]^{\prime}-\mu_{2} N^{2} j_{n}(N \rho)\left[\rho h_{n}^{(1)} \rho\right]^{\prime}}
\end{gathered}
$$

\section{Scattering cross sections}

The ratio of total energy scattered per second, to the incident energy density on a spherical particle, is termed as scattering cross section.

$$
\begin{aligned}
& Q_{s}=\frac{W_{s}}{P_{i}} \\
& Q_{s}=\frac{2 \pi}{k_{2}^{2}} \sum_{n=1}^{\infty}(2 n+1)\left(\left|a_{n}^{r}\right|^{2}+\left|b_{n}^{r}\right|^{2}\right)
\end{aligned}
$$

The mean energy density incident on particle is given by;

$$
\begin{aligned}
& P_{i}=\frac{1}{2} E_{0}^{2} \sqrt{\frac{\varepsilon_{2}}{\mu_{2}}} \\
& W_{s}=\pi \frac{E_{0}^{2}}{k_{2}^{2}} \sqrt{\frac{\varepsilon_{2}}{\mu_{2}}} \sum_{n=1}^{\infty}(2 n+1)\left(\left|a_{n}^{r}\right|^{2}+\left|b_{n}^{r}\right|^{2}\right)
\end{aligned}
$$

The scattered energy can also be found by integrating the Poynting vector over the surface of the sphere under study, which is given by;

$$
\begin{gathered}
W_{\text {ext }}=\iint \mathbf{P}_{\mathbf{s c a}} \cdot \mathbf{n} d S \\
W_{\text {ext }}=\iint\left[\mathbf{E}_{\boldsymbol{s c \boldsymbol { a }}} \cdot \mathbf{H}_{\boldsymbol{s c \boldsymbol { a }}}^{*}\right] \cdot \mathbf{n} d S
\end{gathered}
$$

SCS is calculated using [14];

$$
\sigma_{s c a}=\frac{W_{e x t}}{P_{i n c}}
$$

\section{Extinction /Total cross sections}

The beam of electromagnetic wave is attenuated by absorption and scattering of incident wave. From conservation of energy, we may write;

$$
W_{s}+W_{a}=W_{t}
$$

In terms of cross sections, the above equation may be put in the form,

$$
Q_{s}+Q_{a}=Q_{t}
$$

where,

$$
Q_{t}=\frac{2 \pi}{k_{2}^{2}} \operatorname{Re} \sum_{n=1}^{\infty}(2 n+1)\left(a_{n}^{r}+b_{n}^{r}\right)
$$

\section{Absorption cross sections}

The amount of energy dissipated inside the particle in the form of heat or other forms, is absorbed by the particle. The ratio of energy absorbed by the sphere, to the energy flux density is known as Absorption cross section (ACS).

$$
Q_{a}=Q_{t}-Q_{s}
$$

ACS can also be found by integrating the total heat or energy losses in the particle due to thermal excitations. The rate of energy losses is given by;

$$
\begin{aligned}
& Q_{t}=\frac{2 \pi}{k_{2}^{2}} \operatorname{Re} \sum_{n=1}^{\infty}(2 n+1)\left(a_{n}^{r}+b_{n}^{r}\right) \\
& Q_{\text {loss }}=\frac{1}{2} \operatorname{Re}\left[\mathbf{J}_{t o t} \cdot \mathbf{E}^{*}+j \omega \mathbf{B} \cdot \mathbf{H}^{*}\right]
\end{aligned}
$$

where,

$$
\mathbf{J}_{t o t}=\sigma \mathbf{E}+j \omega \mathbf{D}
$$

i.e. the sum of conduction and displacement currents. The amount of absorbed energy is given by;

$$
W_{a b s}=\iint Q_{l o s s} d V
$$

Finally,

$$
\sigma_{a b s}=\frac{W_{a b s}}{P_{i n}}
$$




\section{Results and discussion}

Fig. 2 shows the scattered efficiencies which has been plotted against the size parameter ' $k a$ '. The complex valued relative permittivity and relative permeability are $\varepsilon_{r}=1.54+0.2 i, \mu_{r}=2(1.54-0.2 i)$ respectively. The parameters for the dielectric sphere are $\varepsilon_{r}=1.54+$ $0.2 i, \mu_{r}=1$, respectively. Solid line shows behavior of dielectric sphere whereas the line with marker shows the behavior of CCM sphere. It can be seen from the plot that the scattered efficiencies are greater in case of CCM sphere than the dielectric sphere. This is due to the reason that imaginary parts of complex valued relative permittivity and permeability gives real valued refractive index as they satisfy the complex conjugate material condition. Hence the losses due to absorption are zero that boost the scattering.

In Fig. 3 the extinction efficiencies have been plotted against the size parameter ' $k a$ '. The values of complex permittivity and permeability are $\varepsilon_{r}=1.54+0.2 \mathrm{i}, \mu_{r}=$ 2(1.54-0.2i) respectively for CCM sphere whereas relative permittivity and relative permeability of dielectric sphere are $\varepsilon_{r}=1.54+0.2 i, \mu_{r}=1$, respectively. Solid line shows extinction due to dielectric sphere whereas the line with marker shows the extinction due to CCM. The extinction due CCM is more than the extinction due to dielectric. It is due to the scattering that contributes more to the extinction.

Figure 4 shows absorption efficiency comparison between CCM sphere and dielectric sphere. Due to complex conjugated values of relative permittivity and permeability, we get real valued refractive index and hence absorption cross section reduced to zero whereas in case of dielectric, the values of relative permittivity and relative permeability are not complex conjugate of each other i.e., $\varepsilon_{r}=1.54+0.2 i, \mu_{r}=1$, as a result we get complex value of refractive index and hence absorption cross section increases. This figure shows that the energy loss of the electromagnetic wave is greater in dielectric than CCM due to absorption.

Figure 5 shows the backscattered efficiencies which have been plotted against the size parameter ' $k a$ '. It can be seen from the graph that the backscattered efficiencies in case of dielectric sphere is almost zero as compared to the CCM sphere. These are the most pronounced and interesting results that can be used in different applications. Taking different values of complex relative permittivity and permeability, we get the same results like in above.

\section{Conclusion}

A sphere made up of complex conjugate medium is considered. Complex conjugate medium is a distinct nature of medium which has real refractive index and complex valued relative permittivity and permeability. When these two parameters $\left(\mu_{r}\right.$ and $\left.\varepsilon_{r}\right)$ become complex conjugate of each other; the refractive index becomes real which makes the medium lossless.

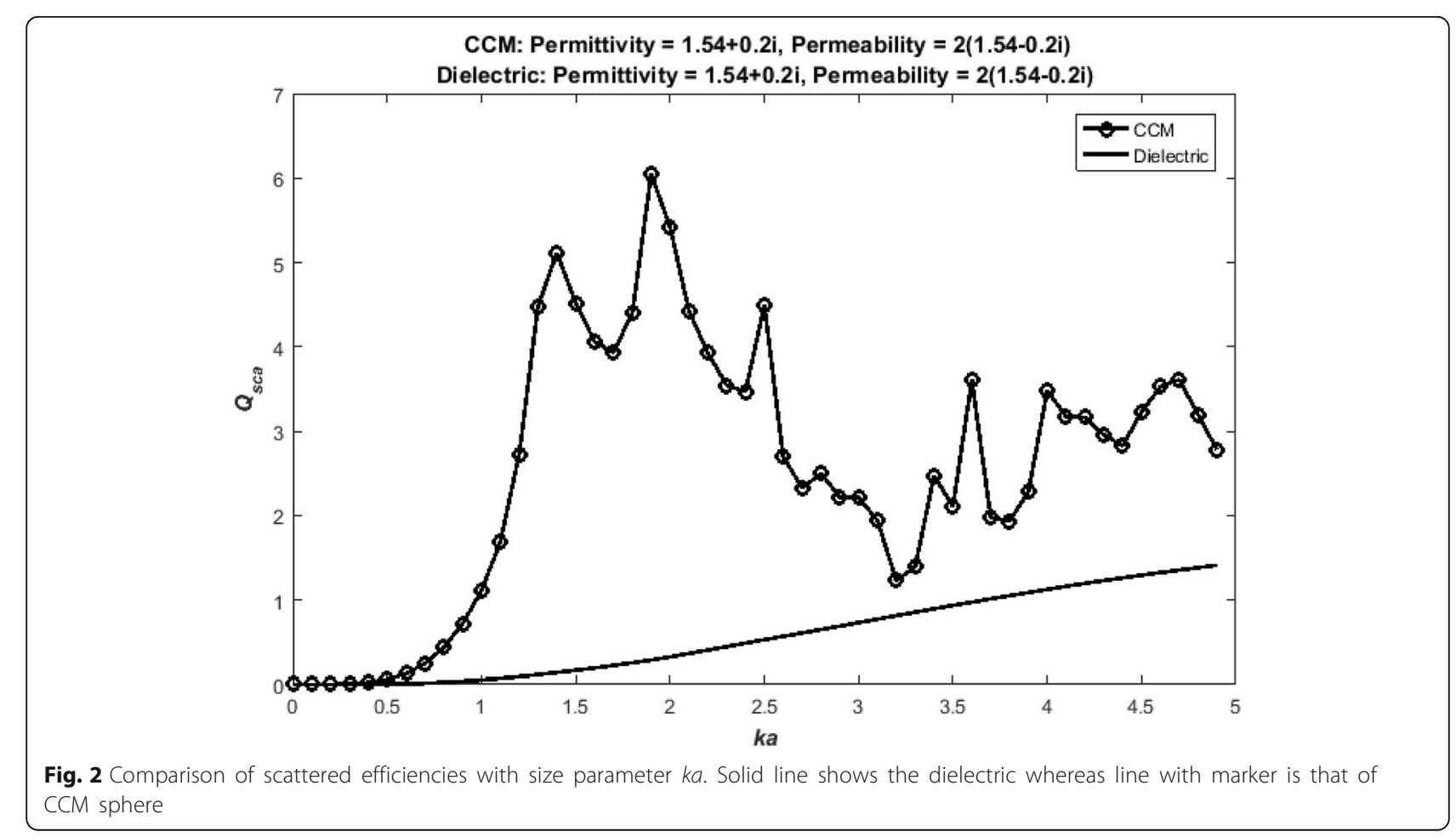




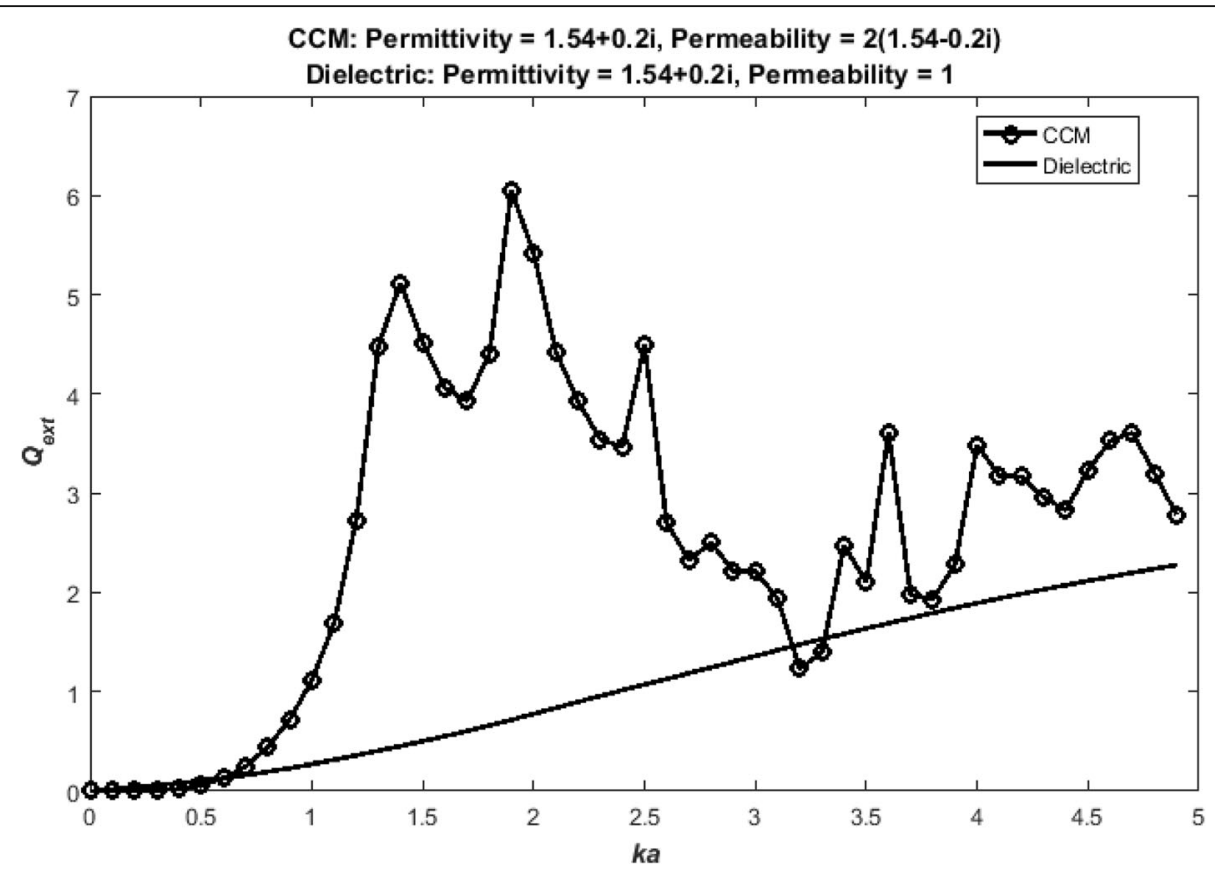

Fig. 3 Comparison of extinction efficiencies with size parameter ka. Solid line shows the dielectric whereas line with marker is that of CCM sphere

These materials have many applications in optics for example optoelectronics, miniaturized amplifiers and lasers etc.

In the present work, the spherical vector wave functions expansion method is used for the analytical result of the problem of the plane wave scattering by CCM sphere. The properties of complex valued constitutive parameters on the scattering properties of a sphere have been investigated. This medium modifies the scattering properties of the sphere. The refractive index of sphere becomes real because the electric permittivity is complex conjugate of the magnetic permeability. Due to the real value of refractive index of this medium the absorption cross section becomes nearly equal to zero and

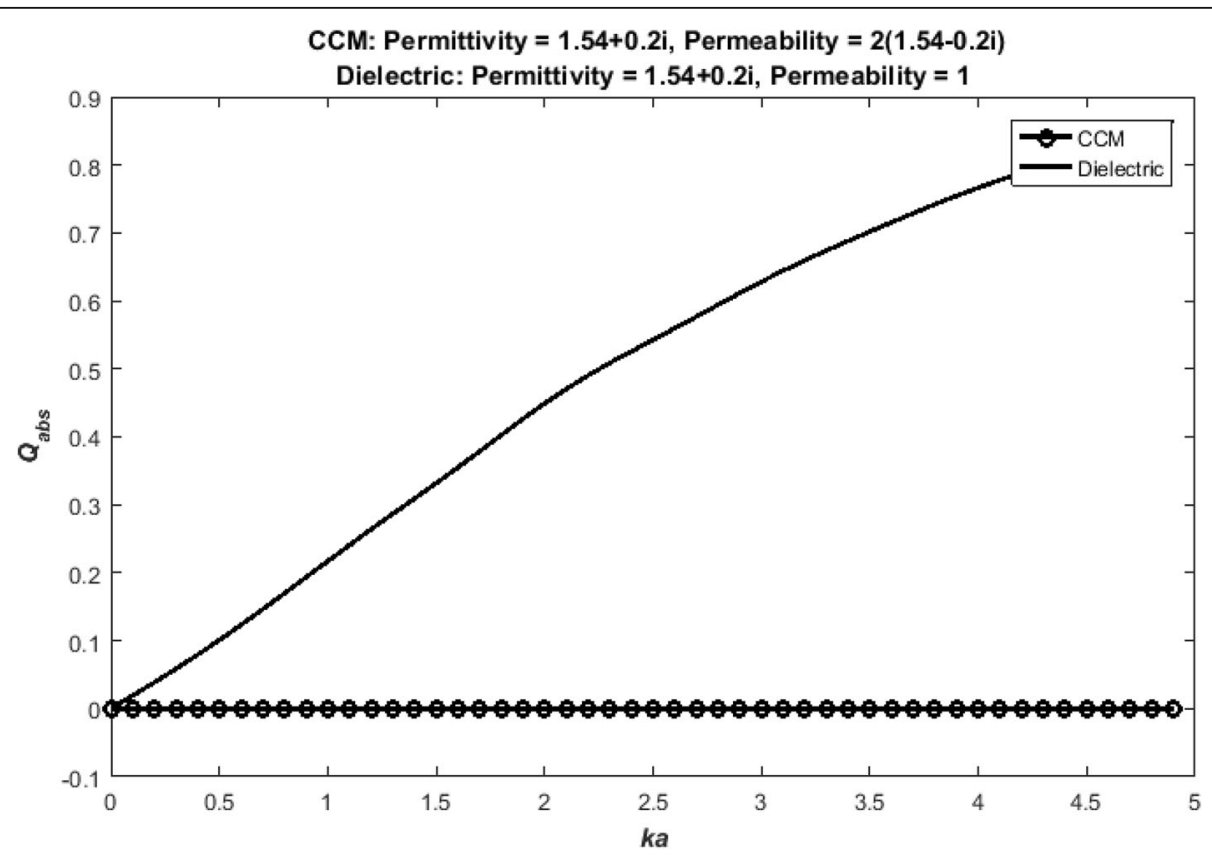

Fig. 4 Comparison of extinction efficiencies with size parameter $k a$. Solid line shows the dielectric whereas line with marker is that of CCM sphere 
CCM: Permittivity = 1.54+0.2i, Permeability $=2(1.54-0.2 i)$

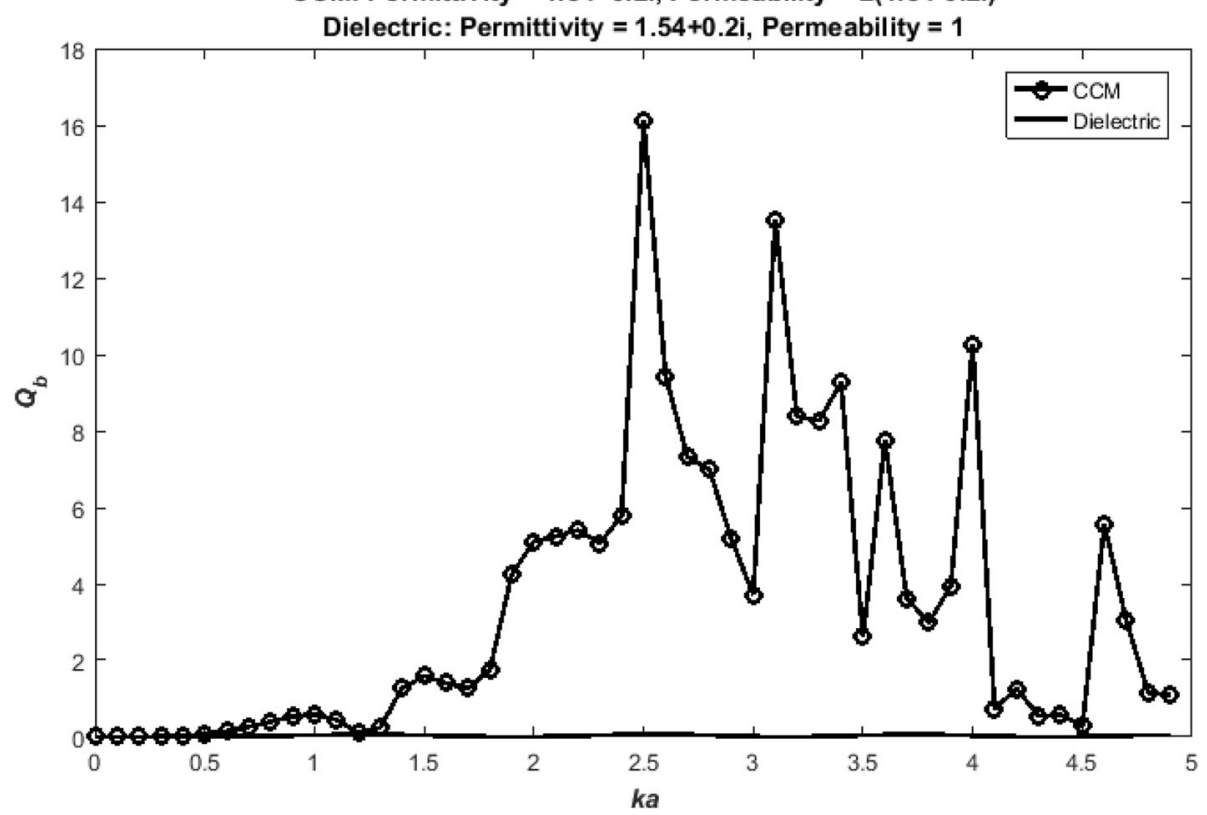

Fig. 5 Comparison of backscattered efficiencies with size parameter ka. Solid line shows the dielectric whereas line with marker is that of CCM sphere

extinction will be only due to the scattering. In other words, the scattered field is only due to the impedance mismatch at the boundary of the sphere. Mie efficiencies of dielectric and CCM sphere have been plotted against the size parameter of the sphere and found that the scattering in case of CCM sphere is dominating over that of dielectric case. Arrays of CCM spheres may be used as a promising light trapping schemes for thin film solar cells.

\section{Acknowledgements}

Not applicable.

\section{Authors' contributions}

Al, MAG, MA derived analytical expressions and numerical analysis. They wrote the main manuscript text. SK and YK developed methodology in the given study. All authors read and approved the final manuscript.

\section{Funding}

Deanship of Scientific Research (DSR) at King Saud University for its funding of this research through the Research Group no RG-1436-012.

\section{Availability of data and materials}

Detail about data has been provided in the article.

\section{Competing interests}

The authors declare that they have no competing interests.

\section{Author details}

'Department of Physics, Research in Modeling and Simulation (RIMS) Group, COMSATS University Islamabad, Islamabad, Pakistan. ${ }^{2}$ Department of Electrical Engineering, University of Management and Technology, Lahore, Pakistan. ${ }^{3}$ Department of Electrical Engineering, King Saud University, Riyadh, Saudi Arabia. ${ }^{4}$ Department of Physics, University of Agriculture Faisalabad, Faisalabad, Pakistan.
Received: 28 December 2018 Accepted: 20 May 2019 Published online: 07 June 2019

\section{References}

1. Dragoman, D.: Complex conjugate media: alternative configurations for miniaturized lasers. Opt. Commun. 284, 2095-2098 (2011)

2. Rao, S., Wilton, D., Glisson, A.: Electromagnetic scattering by surfaces of arbitrary shape. IEEE Trans. Antennas Propag. 30, 409-418 (1982)

3. Bin-Jie, H., Kai-Ning, E.Y., Jun, Z., Serge, T.: Scattering characteristics of conducting cylinder coated with nonuniform magnetized ferrite. Chin. Phys. 14, 2305 (2005)

4. Sadrara, M., Miri, M.: Scattering of electromagnetic waves by a cluster of charged spherical nanoparticles. JOSA B. 33, 2552-2559 (2016)

5. Xiang, J., He, S., Zhang, Y., Zhu, G.: A spectral domain approach for the calculation of the scattering of the stratified uniaxial electric anisotropic media under point source excitation with arbitrary orientation. Int. J. Appl. Electromagn. Mech. 48, 33-46 (2015)

6. Hamid, A., Cooray, F.: TE scattering by a perfect electromagnetic conducting semi-elliptic-cylindrical boss on a perfectly conducting plane. Int. J. Appl. Electromagn. Mech. 38, 1-8 (2012)

7. Zimmermann, E., Dändliker, R., Souli, N., Krattiger, B.: Scattering of an off-axis Gaussian beam by a dielectric cylinder compared with a rigorous electromagnetic approach. JOSA A. 12, 398-403 (1995)

8. Lock, J.A.: Scattering of a diagonally incident focused Gaussian beam by an infinitely long homogeneous circular cylinder. JOSA A. 14, 640-652 (1997)

9. Tzarouchis, D., Sihvola, A.: Light scattering by a dielectric sphere: perspectives on the Mie resonances. Appl. Sci. 8, 184 (2018)

10. Lakhtakia, A., Mackay, T.G.: Electromagnetic scattering by homogeneous, isotropic, dielectric-magnetic sphere with topologically insulating surface states. JOSA B. 33, 603-609 (2016)

11. Yang, Z., Gao, P., Zhang, C., Li, X., Ye, J.: Scattering effect of the high-index dielectric nanospheres for high performance hydrogenated amorphous silicon thin-film solar cells. Sci. Rep. 6, 30503 (2016)

12. Spinelli, P., Lenzmann, F., Weeber, A., Polman, A.: Effect of EVA encapsulation on antireflection properties of Mie nanoscatterers for c-Si solar cells. IEEE $J$. Photovolt. 5, 559-564 (2015) 
13. Stratton, J.A.: Electromagnetic Theory. McGraw-Hill Comp., New York and London (1941).

14. Yushanov, S., Crompton, J.S., Koppenhoefer, K.C.: Mie Scattering of Electromagnetic Waves, in Proc. COMSOL conf, pp. 1-7 (2013)

\section{Publisher's Note}

Springer Nature remains neutral with regard to jurisdictional claims in published maps and institutional affiliations.

Submit your manuscript to a SpringerOpen ${ }^{\mathcal{O}}$ journal and benefit from:

- Convenient online submission

- Rigorous peer review

- Open access: articles freely available online

High visibility within the field

- Retaining the copyright to your article

Submit your next manuscript at $\boldsymbol{\nabla}$ springeropen.com 